\title{
Solutions of a Quadratic Inverse Eigenvalue Problem for Damped Gyroscopic Second-Order Systems
}

\author{
Hong-Xiu Zhong, Guo-Liang Chen, and Xiang-Yun Zhang \\ Department of Mathematics, East China Normal University, Shanghai 200241, China \\ Correspondence should be addressed to Guo-Liang Chen; glchen@math.ecnu.edu.cn
}

Received 2 September 2013; Accepted 15 December 2013; Published 21 January 2014

Academic Editor: Zhongxiao Jia

Copyright ( 2014 Hong-Xiu Zhong et al. This is an open access article distributed under the Creative Commons Attribution License, which permits unrestricted use, distribution, and reproduction in any medium, provided the original work is properly cited.

Given $k$ pairs of complex numbers and vectors (closed under conjugation), we consider the inverse quadratic eigenvalue problem of constructing $n \times n$ real matrices $M, D, G$, and $K$, where $M>0, K$ and $D$ are symmetric, and $G$ is skew-symmetric, so that the quadratic pencil $Q(\lambda)=\lambda^{2} M+\lambda(D+G)+K$ has the given $k$ pairs as eigenpairs. First, we construct a general solution to this problem with $k \leq n$. Then, with the special properties $D=0$ and $K<0$, we construct a particular solution. Numerical results illustrate these solutions.

\section{Introduction}

Vibrating structures such as buildings, bridges, highways, and airplanes are distributed parameter systems [1]. Very often a distributed parameter system is first discretized to a matrix second-order using techniques of finite element or finite difference, and then an approximate solution is obtained for the discretized model. Associated with the matrix second-order model is the eigenvalue problem of the quadratic pencil,

$$
Q(\lambda) \equiv \lambda^{2} M+\lambda(D+G)+K,
$$

where $M, D, G$, and $K$ are, respectively, mass, damping, gyroscopic and stiffness matrices.

The system represented by (1) is called damped gyroscopic system. In general, the gyroscopic matrix $G$ is always skew-symmetric, the damping matrix $D$ and the stiffness matrix $K$ are symmetric, the mass matrix $M$ is symmetric positive definite, and they are all $n \times n$ real matrices. If $G=0$, the system is called damped nongyroscopic system, and if $D=0$, the system is called undamped gyroscopic system.

The damped gyroscopic system has been widely studied in two aspects: the quadratic eigenvalue problem (QEP) and the quadratic inverse eigenvalue problem (QIEP). The QEP involves finding scalars $\lambda \in \mathbb{C}$ and nonzero vectors $\mathbf{x} \in \mathbb{C}^{n}$, called the eigenvalues and eigenvectors of the system, to satisfy the algebraic equation $Q(\lambda) \mathbf{x}=0$, when the coefficient matrices are given. Many authors have been devoted to this kind of problems and a series of good results have been made (see, e.g., [2-8]). The QIEP determines or estimates the parameters of the system from observed or expected eigeninformation of $Q(\lambda)$. Our main interest in this paper is the corresponding inverse problem: given partially measured information about eigenvalues and eigenvectors, we reconstruct matrices $M, D, G$, and $K$, satisfied with several conditions, so that $Q(\lambda)$ has the given $k$ eigenpairs. The problem we considered is stated as follows.

Problem 1. Given an eigeninformation pair $(\Lambda, X) \in \mathbb{R}^{k \times k} \times$ $\mathbb{R}^{n \times k}(k \leq n)$, where

$$
\Lambda=\operatorname{diag}\left\{\lambda_{1}^{[2]}, \ldots, \lambda_{\ell}^{[2]}, \lambda_{2 \ell+1}, \ldots, \lambda_{k}\right\},
$$

with

$$
\begin{gathered}
\lambda_{j}^{[2]}=\left[\begin{array}{cc}
\alpha_{j} & \beta_{j} \\
-\beta_{j} & \alpha_{j}
\end{array}\right] \in \mathbb{R}^{2 \times 2}, \quad \beta_{j} \neq 0, \text { for } j=1, \ldots, \ell, \\
X=\left[\mathbf{x}_{1 R}, \mathbf{x}_{1 I}, \ldots, \mathbf{x}_{\ell R}, \mathbf{x}_{\ell I} ; \mathbf{x}_{2 \ell+1}, \ldots, \mathbf{x}_{k}\right],
\end{gathered}
$$

find $n \times n$ real matrices $M, D, G$, and $K$, with $M$ being symmetric definite, $D$ and $K$ being symmetric, and $G$ being skew-symmetric, so that

$$
M X \Lambda^{2}+(D+G) X \Lambda+K X=0 .
$$


In [9], Gohberg et al. developed a powerful GLR theory to solve the QIEP of the undamped gyroscopic system. In [10], $\mathrm{Chu}$ and $\mathrm{Xu}$ developed an elegant procedure to obtain a realvalued spectral decomposition of the damped nongyroscopic system. And then, Jia and Wei [11] derived a real-valued spectral decomposition of the undamped gyroscopic system. However, $[10,11]$ both need all the eigen-information of $Q(\lambda)$ to obtain the parameters, and it is often impractical or impossible to obtain complete spectral information. Thus, it becomes very interesting to consider a QIEP with only a subset of eigenpairs known.

In [12], Kuo et al. constructed the solutions of the QIEP of the damped nongyroscopic system with $k \leq n$ given eigenpairs. And for the same system, Cai et al. [13] solved the QIEP with $2 n \geq k>n$ given eigenpairs. Meanwhile, for the damped gyroscopic system, Yuan [14] solved the QIEP with $k$ given eigenpairs. In [14], Yuan constructed symmetric positive semidefinite matrix $D$ and skew-symmetric matrix $G$ for $Q(\lambda)$, with $M>0$ and $K \geq 0$ as given matrices. So, it becomes challenging to construct $(M, D, G, K)$ for damped gyroscopic system (1) with $k$ given eigenpairs, and this is the goal of this paper.

This paper is organized as follows. In Section 2, we establish the solubility theory of the Problem 1. In Section 3, we develop a simple method to compute a particular solution to the Problem 1 with $D=0$. Moreover, for $D=0$ and $K<0$, a simple algorithm is developed to compute a solution in Section 4. Some numerical results are presented in Section 5 to illustrate our main results. In the last section, some conclusions and acknowledgements are given.

Throughout this paper, we use capital letters to denote matrices, and lowercase (bold) letters to denote scalars (vectors). $A^{T}$ denotes the transpose of the matrix $A, I_{n}$ denotes the $n \times n$ identity matrix, and $A^{\dagger}$ denotes the Moore-Penrose generalized inverse of $A$. We write $A>0(A \geq 0)$ if $A$ is real symmetric positive (semidefinite). The spectrum of $A$ is denoted by $\sigma(A)$.

For simplicity, we make the following assumptions.

(A1) The eigenvector matrix $X$ in Problem 1 has full column rank, that is, $\operatorname{rank}(X)=k$.

(A2) The eigenvalue matrix $\Lambda$ in Problem 1 has only simple eigenvalues, that is, $0 \notin \sigma(\Lambda)$.

Remark 2. For the case that $0 \in \sigma(\Lambda)$, using the assumption of simple eigenvalues, we can partition $\Lambda=\operatorname{diag}\left\{\Lambda_{1}, 0\right\}$, where $\Lambda_{1}$ has no zero eigenvalue, and then do discussion with $\Lambda_{1}$. So, in this paper, we only consider the case that $\Lambda$ has no zero eigenvalue.

\section{General Solution of the Problem}

In this section, we will give a general solution to the Problem 1 for given matrix pair $(\Lambda, X) \in \mathbb{R}^{k \times k} \times \mathbb{R}^{n \times k}(k \leq n)$ as in (2) and (3). At the beginning, we will introduce some lemmas.

Lemma 3 (see [15]). Let $A \in \mathbb{R}^{m \times \ell}$ and $B \in \mathbb{R}^{\ell \times \ell}$; then

$$
A^{T} X-X^{T} A=B
$$

has a solution $X \in \mathbb{R}^{m \times \ell}$ if and only if

$$
B^{T}=-B, \quad\left(I_{\ell}-A^{\dagger} A\right) B\left(I_{\ell}-A^{\dagger} A\right)=0,
$$

where $A^{\dagger}$ is the Moore-Penrose generalized inverse of $A$.

When condition (6) is satisfied, the general solution of (5) is

$$
\begin{aligned}
X= & \frac{1}{2}\left(A^{\dagger}\right)^{T} B A^{\dagger} A+\left(A^{\dagger}\right)^{T} B\left(I_{\ell}-A^{\dagger} A\right) \\
& +\left(I_{m}-A A^{\dagger}\right) Y+A A^{\dagger} Z A,
\end{aligned}
$$

where $Y \in \mathbb{R}^{m \times \ell}$ is arbitrary and $Z \in \mathbb{R}^{m \times m}$ is constrained only by the symmetry requirement that

$$
\left(A A^{\dagger} Z A A^{\dagger}\right)^{T}=A A^{\dagger} Z A A^{\dagger}
$$

Lemma 3 directly results in the following lemma.

Lemma 4. Let $A \in \mathbb{R}^{m \times m}$ be a nonsingular matrix and $B \in$ $\mathbb{R}^{m \times m}$; then

$$
A^{T} X-X^{T} A=B
$$

has a solution $X \in \mathbb{R}^{m \times m}$ if and only if

$$
B^{T}=-B
$$

in which case the general solution is $X=(1 / 2) A^{-T} B+Z A$, where $Z=Z^{T} \in \mathbb{R}^{m \times m}$ is an arbitrary matrix.

Given matrix pair $(\Lambda, X) \in \mathbb{R}^{k \times k} \times \mathbb{R}^{n \times k}(k \leq n)$ as in Problem 1: let

$$
X=Q\left[\begin{array}{l}
R \\
0
\end{array}\right]
$$

be the $Q R$-factorization of $X$, where $Q \in \mathbb{R}^{n \times n}$ is orthogonal and $R \in \mathbb{R}^{k \times k}$ is upper triangular. We may require that $R$ has positive diagonal entries, since $X$ is of full column rank.

Let $C=D+G$, so that finding $M, D, G$, and $K$ which satisfy (4) is equivalent to finding $M, C$, and $K$ which satisfy

$$
M X \Lambda^{2}+C X \Lambda+K X=0,
$$

and the relations of $C, D$, and $G$ are

$$
D=\frac{1}{2}\left(C+C^{T}\right), \quad G=\frac{1}{2}\left(C-C^{T}\right) .
$$

Let $S=R \Lambda R^{-1}$; we can see $S^{-1}$ exists by using $0 \notin \sigma(\Lambda)$. Denoting

$$
\begin{gathered}
M_{\mathrm{Q}}=Q^{T} M Q=\left[\begin{array}{ll}
M_{11} & M_{12} \\
M_{21} & M_{22}
\end{array}\right], \\
C_{\mathrm{Q}}=Q^{T} C Q=\left[\begin{array}{ll}
C_{11} & C_{12} \\
C_{21} & C_{22}
\end{array}\right], \\
K_{\mathrm{Q}}=Q^{T} K Q=\left[\begin{array}{ll}
K_{11} & K_{12} \\
K_{21} & K_{22}
\end{array}\right],
\end{gathered}
$$

where $M_{11}, C_{11}, K_{11} \in \mathbb{R}^{k \times k}, M_{12}=M_{21}^{T}, K_{12}=K_{21}^{T}, C_{12}$, $C_{21}^{T} \in \mathbb{R}^{k \times(n-k)}, M_{22}, C_{22}, K_{22} \in \mathbb{R}^{(n-k) \times(n-k)}$, we will obtain the following main theorem. 
Theorem 5. Let $M_{Q}, C_{Q}$, and $K_{Q}$ be defined as in (14)-(16); then there are real matrices $M, C=D+G$ and $K$ satisfy (4) if and only if

(i) $\left[\begin{array}{ll}M_{11} & M_{12} \\ M_{21} & M_{22}\end{array}\right]$ is arbitrarily symmetric positive definite,

(ii) $C_{12}, C_{21}, C_{22}$ are arbitrary,

(iii) $K_{22}=K_{22}^{T}$ is arbitrary symmetric,

(iv) $C_{11}=(1 / 2)\left(S^{2}\right)^{T} M_{11} S^{-1}-(1 / 2) M_{11} S+S^{T} Z$, where $Z \in \mathbb{R}^{k \times k}$ is arbitrary symmetric,

(v) $K_{11}=K_{11}^{T}=-\left((1 / 2)\left(S^{2}\right)^{T} M_{11}+(1 / 2) M_{11} S^{2}+S^{T} Z S\right)$,

(vi) $K_{21}=K_{12}^{T}=-\left(M_{21} S^{2}+C_{21} S\right)$.

Furthermore, $D$ and $G$ can be expressed as in (13).

Proof. Necessity. From (14)-(16), we know $M=Q M_{\mathrm{Q}} Q^{T}, C=$ $Q C_{\mathrm{Q}} Q^{T}$, and $K=Q K_{\mathrm{Q}} Q^{T}$; substituting them and (11) into (12), we have

$$
\begin{aligned}
& M_{11} S^{2}+C_{11} S+K_{11}=0, \\
& M_{21} S^{2}+C_{21} S+K_{21}=0 .
\end{aligned}
$$

Thus, finding $M, C$, and $K$ which satisfy (12) is equivalent to finding the submatrices $M_{11}, M_{21}, C_{11}, C_{21}, K_{11}$, and $K_{21}$ which satisfy (17) and (18). Clearly, it follows from (18) that $K_{21}$ is determined by

$$
K_{21}=K_{12}^{T}=-\left(M_{21} S^{2}+C_{21} S\right),
$$

where $M_{21}$ and $C_{21}$ are arbitrary.

As $M$ and $K$ are required to be symmetric positive definite and symmetric, respectively, so are $M_{11}$ and $K_{11}$ in (14) and (16). From (17) it follows that

$$
K_{11}=-M_{11} S^{2}-C_{11} S \text {. }
$$

Let $M_{11}$ be an arbitrary symmetric positive definite matrix. We need to find $C_{11}$ such that $K_{11}$ is symmetric; that is, it satisfies

$$
\left(M_{11} S^{2}+C_{11} S\right)^{T}=M_{11} S^{2}+C_{11} S .
$$

After rearrangement, (21) becomes

$$
S^{T} C_{11}^{T}-C_{11} S=M_{11} S^{2}-\left(S^{2}\right)^{T} M_{11} .
$$

Because $\left(M_{11} S^{2}-\left(S^{2}\right)^{T} M_{11}\right)^{T}=-\left(M_{11} S^{2}-\left(S^{2}\right)^{T} M_{11}\right)$ and $S$ is nonsingular, we can get from Lemma 4 that

$$
C_{11}=\frac{1}{2}\left(S^{2}\right)^{T} M_{11} S^{-1}-\frac{1}{2} M_{11} S+S^{T} Z,
$$

where $Z \in \mathbb{R}^{k \times k}$ is arbitrary symmetric. Substituting (23) into (20) yields (v). Furthermore, $D$ and $G$ can be expressed as in (13).

Sufficiency. From the description of (i)-(vi), we can obtain that (12) holds; thus (4) holds with

$$
D=\frac{1}{2}\left(C+C^{T}\right), \quad G=\frac{1}{2}\left(C-C^{T}\right) .
$$

Remark 6. The general solution to the Problem 1 with $G=0$ and $(k \leq n)$ prescribed eigenpairs has been given in [12], here, we generalize its solution to the case of $G \neq 0$.

Remark 7. It is complicated for the more general case $\operatorname{rank}(X)<k$, and we will discuss it in our next work. However, here we provide a simple solution. We can select the linear independent columns and the relevant eigenvalues to construct a new $X^{\prime}$ and $\Lambda^{\prime}$, then do discussion with them.

Remark 8 . When $k=n$, by Theorem 5 , the general solution of the Problem 1 is given by

$$
M=Q M_{Q} Q^{T}, \quad C=Q C_{Q} Q^{T}, \quad K=Q K_{G} Q^{T},
$$

where

$$
\begin{gathered}
C_{\mathrm{Q}}=\frac{1}{2}\left(S^{2}\right)^{T} M_{\mathrm{Q}} S^{-1}-\frac{1}{2} M_{\mathrm{Q}} S+S^{T} Z, \\
K_{\mathrm{Q}}=-\left(\frac{1}{2}\left(S^{2}\right)^{T} M_{\mathrm{Q}}+\frac{1}{2} M_{\mathrm{Q}} S^{2}+S^{T} Z S\right),
\end{gathered}
$$

with $M_{\mathrm{Q}}>0$ and $M_{\mathrm{Q}}=M_{\mathrm{Q}}^{T}$ which can be arbitrarily chosen and $Z \in \widehat{R}^{n \times n}$ is arbitrarily symmetric.

Using Theorem 5, we can construct a solution to the Problem 1 as follows.

Algorithm 9. An algorithm for solving Problem 1 is proposed as follows.

(1) Input $\Lambda$ and $X$, compute the $Q R$ decomposition of $X$ according to (11), and compute $S=R \Lambda R^{-1}$.

(2) Choose a symmetric positive definite matrix $M_{11}$ and a symmetric matrix $Z$, arbitrarily. Compute $C_{11}$ and $K_{11}$ by (iv) and (v) in Theorem 5 , respectively.

(3) Choose arbitrary $M_{21}$ and $C_{21}$, and compute $K_{21}$ by (vi) in Theorem 5, $M_{12}=M_{21}^{T}$ and $K_{12}=K_{21}^{T}$.

(4) Choose a symmetric positive definite matrix $\widehat{M} \in$ $\mathbb{R}^{(n-k) \times(n-k)}$; compute $M_{22}=\widehat{M}+M_{21} M_{11}^{-1} M_{21}^{T}$.

(5) Choose arbitrary matrices $C_{12}$ and $C_{22}$ and a symmetric matrix $K_{22}$, and form

$$
M=Q M_{Q} Q^{T}, \quad C=Q C_{Q} Q^{T}, \quad K=Q K_{Q} Q^{T},
$$

where $Q$ is given by (11). Compute $D$ and $G$ by (13).

\section{Particular Solutions with $D=0$}

As we all know, the applications of the undamped gyroscopic system (i.e., $D=0$ ) exist in many fields; for details, see [5]. In this section, we will discuss the particular solutions of Problem 1 with $D=0$ and $(k \leq n)$ prescribed eigenpairs. And in this case, $Q(\lambda)$ in (1) becomes

$$
Q(\lambda) \equiv \lambda^{2} M+\lambda G+K .
$$


It is well known that the eigenvalues of (28) have a Hamiltonian structure; that is, they occur in quadruples $(\lambda, \bar{\lambda},-\bar{\lambda}$, $-\lambda$ ), possibly collapsing to real or imaginary pairs or singlezero eigenvalues. In [11], Jia and Wei discussed the eigenvalues of $Q(\lambda)$ in (28) and separated them into four categories. From the assumption (A2), we can know $k$ is even. Here we rewrite the given eigeninformation pair $(\Lambda, X)$ of Problem 1 as

$$
\Lambda=\operatorname{diag}\left\{\Lambda_{1}, \Lambda_{2}, \ldots, \Lambda_{\ell_{3}}\right\},
$$

with

$$
\begin{gathered}
\Lambda_{j}=\operatorname{diag}\left\{\left[\begin{array}{cc}
\alpha_{j} & \beta_{j} \\
-\beta_{j} & \alpha_{j}
\end{array}\right],\left[\begin{array}{cc}
-\alpha_{j} & \beta_{j} \\
-\beta_{j} & -\alpha_{j}
\end{array}\right]\right\} \in \mathbb{R}^{4 \times 4}, \\
\alpha_{j}>0, \beta_{j}>0, j=1, \ldots, \ell_{1}, \\
\Lambda_{j}=\left[\begin{array}{cc}
0 & \beta_{j} \\
-\beta_{j} & 0
\end{array}\right] \in \mathbb{R}^{2 \times 2}, \quad \beta_{j}>0, j=\ell_{1}+1, \ldots, \ell_{2}, \\
\Lambda_{j}=\left[\begin{array}{cc}
\alpha_{j} & 0 \\
0 & -\alpha_{j}
\end{array}\right] \in \mathbb{R}^{2 \times 2}, \quad \alpha_{j}>0, j=\ell_{2}+1, \ldots, \ell_{3}, \\
X=\left[\begin{array}{c}
\mathbf{x}_{1 R^{+}}, \mathbf{x}_{1 I^{+}}, \mathbf{x}_{1 R^{-}}, \mathbf{x}_{1 I^{-}}, \ldots, \mathbf{x}_{\ell_{1} R^{+}}, \mathbf{x}_{\ell_{1} I^{+}}, \mathbf{x}_{\ell_{1} R^{-}}, \mathbf{x}_{\ell_{1} I^{-}} ; \\
\mathbf{x}_{\left(\ell_{1}+1\right) R}, \mathbf{x}_{\left(\ell_{1}+1\right) I}, \ldots, \mathbf{x}_{\ell_{2} R^{2}}, \mathbf{x}_{\ell_{2} I} ; \\
\mathbf{x}_{\left(\ell_{2}+1\right) R^{+}}, \mathbf{x}_{\left(\ell_{2}+1\right) R^{-}}, \ldots, \mathbf{x}_{\ell_{3} R^{+}}, \mathbf{x}_{\ell_{3} R^{-}}
\end{array}\right],
\end{gathered}
$$

where

$$
\begin{gathered}
{\left[\mathbf{x}_{j R^{+}} \pm i \mathbf{x}_{j I^{+}}, \alpha_{j} \pm i \beta_{j}\right],} \\
{\left[\mathbf{x}_{j R^{-}} \pm i \mathbf{x}_{j I^{-}},-\alpha_{j} \pm i \beta_{j}\right]} \\
j=1, \ldots, \ell_{1} ; \\
{\left[\mathbf{x}_{j R} \pm i \mathbf{x}_{j I}, \pm i \beta_{j}\right], \quad j=\ell_{1}+1, \ldots, \ell_{2} ;} \\
{\left[\mathbf{x}_{j R^{ \pm}}, \pm \alpha_{j}\right], \quad j=\ell_{2}+1, \ldots, \ell_{3},}
\end{gathered}
$$

are eigenpairs, and $4 \ell_{1}+2\left(\ell_{2}-\ell_{1}\right)+2\left(\ell_{3}-\ell_{2}\right)=2 \ell_{1}+2 \ell_{3}=k$.

In this section, the Problem 1 becomes the following problem.

Given an eigeninformation pair $(\Lambda, X)(k \leq n)$ with (29)(31), find $n \times n$ real matrices $M, G$, and $K$, with $M$ being symmetric definite, $K$ being symmetric, and $G$ being skewsymmetric, so that,

$$
M X \Lambda^{2}+G X \Lambda+K X=0 .
$$

As well as in Section 2, let

$$
G_{Q}=Q^{T} G Q=\left[\begin{array}{ll}
G_{11} & G_{12} \\
G_{21} & G_{22}
\end{array}\right],
$$

where $G_{Q}$ is partitioned conforming with that of $M$ in (14), and we can easily calculate that $G$ also satisfies the action of $C$ in Section 2, except that $G$ has an additional property, that is, $G^{T}=-G$. In the following theorem, we will discuss the solubility of Problem 1 with $D=0$ and $k \leq n$.
Theorem 10. Let $M_{\mathrm{Q}}, K_{\mathrm{Q}}$, and $G_{\mathrm{Q}}$ be defined as in (14), (16) and (34); then there are real matrices $M, G$, and $K$ which satisfy (33) if and only if

(i) $\left[\begin{array}{ll}M_{11} & M_{12} \\ M_{21} & M_{22}\end{array}\right]$ is arbitrarily symmetric positive definite,

(ii) $G_{12}=-G_{21}^{T}$ and $G_{22}=-G_{22}^{T}$ are arbitrary,

(iii) $K_{22}=K_{22}^{T}$ is arbitrary symmetric,

(iv) $G_{11}=-G_{11}=-R^{-T} \Gamma R^{-1}-M_{11} S+S^{T} M_{11}$,

(v) $K_{11}=K_{11}^{T}=R^{-T} \Gamma \Lambda R^{-1}-S^{T} M_{11} S$,

(vi) $K_{21}=K_{12}^{T}=-\left(M_{21} S^{2}+G_{21} S\right)$,

in which

$$
\begin{aligned}
& \Gamma=\operatorname{diag}\left\{\begin{array}{cccc}
0 & 0 & \xi_{1} & \eta_{1} \\
0 & 0 & -\eta_{1} & \xi_{1} \\
-\xi_{1} & \eta_{1} & 0 & 0 \\
-\eta_{1} & -\xi_{1} & 0 & 0
\end{array}\right], \ldots, \\
& {\left[\begin{array}{cccc}
0 & 0 & \xi_{\ell_{1}} & \eta_{\ell_{1}} \\
0 & 0 & -\eta_{\ell_{1}} & \xi_{\ell_{1}} \\
-\xi_{\ell_{1}} & \eta_{\ell_{1}} & 0 & 0 \\
-\eta_{\ell_{1}} & -\xi_{\ell_{1}} & 0 & 0
\end{array}\right], } \\
& {\left.\left[\begin{array}{cc}
0 & \eta_{\ell_{1}+1} \\
-\eta_{\ell_{1}+1} & 0
\end{array}\right], \ldots,\left[\begin{array}{cc}
0 & \eta_{\ell_{3}} \\
-\eta_{\ell_{3}} & 0
\end{array}\right]\right\}, }
\end{aligned}
$$

with $\xi_{j}$ and $\eta_{j}$ being arbitrary real numbers.

Proof. Necessity. Same as the proof of Theorem 5, we can get

$$
K_{21}=K_{12}^{T}=-\left(M_{21} S^{2}+G_{21} S\right),
$$

where $M_{21}$ and $G_{21}$ are arbitrary. We also have

$$
K_{11}=-M_{11} S^{2}-G_{11} S,
$$

and $G_{11}$ satisfies

$$
\left(M_{11} S^{2}+G_{11} S\right)^{T}=M_{11} S^{2}+G_{11} S .
$$

After rearrangement, (38) becomes

$$
G_{11} S+S^{T} G_{11}=-M_{11} S^{2}+\left(S^{2}\right)^{T} M_{11} .
$$

It is easily seen that (39) has a particular solution

$$
G_{11}^{(0)}=-M_{11} S+S^{T} M_{11} .
$$

Next, we consider the homogeneous equation

$$
G_{11} S+S^{T} G_{11}=0
$$

Substituting $S=R \Lambda S^{-1}$ into (41), we get

$$
\left(R^{T} G_{11} R\right) \Lambda+\Lambda^{T}\left(R^{T} G_{11} R\right)=0 .
$$


Write $\Gamma \equiv R^{T} G_{11} R=\left(\Gamma_{m j}\right)$, where $\Gamma$ is partitioned conforming with that of $\Lambda$ in (29). Then we observe that

$$
\Gamma_{m j} \Lambda_{j}+\Lambda_{m}^{T} \Gamma_{m j}=0, \quad m, j=1, \ldots, \ell_{3} .
$$

When $m \neq j$, (43) can be rewritten as

$$
\left[\left(\Lambda_{j}^{T}\right) \otimes I+I \otimes\left(\Lambda_{m}^{T}\right)\right] \operatorname{vec}\left(\Gamma_{m j}\right)=0,
$$

in which $\otimes$ stands for the Kronecker product and vec stands for the column vectorization of a matrix. Because $m \neq j$, and assumption (A2), we know that $\left(\Lambda_{j}^{T}\right) \otimes I+I \otimes\left(\Lambda_{m}^{T}\right)$ is nonsingular; therefore $\operatorname{vec}\left(\Gamma_{m j}\right)=0$, so $\Gamma_{m j}=0$.

Now we discuss the structures of matrices $\Gamma_{j j}$, $j=$ $1, \ldots, \ell_{3}$, which are skew-symmetric. For simplicity, we denote $\Gamma_{j j}$ by $\Gamma_{j}$. Then we need to solve

$$
\Gamma_{j} \Lambda_{j}+\Lambda_{j}^{T} \Gamma_{j}=0, \quad j=1, \ldots, \ell_{3} .
$$

Since $\Lambda_{j}$ has the form in (30), we can easily compute that the general solution of (45) has the form

$$
\begin{gathered}
\Gamma_{j}=\left[\begin{array}{cccc}
0 & 0 & \xi_{j} & \eta_{j} \\
0 & 0 & -\eta_{j} & \xi_{j} \\
-\xi_{j} & \eta_{j} & 0 & 0 \\
-\eta_{j} & -\xi_{j} & 0 & 0
\end{array}\right], \quad j=1, \ldots, \ell_{1}, \\
\Gamma_{j}=\left[\begin{array}{cc}
0 & \eta_{j} \\
-\eta_{j} & 0
\end{array}\right], \quad j=\ell_{1}+1, \ldots, \ell_{3} .
\end{gathered}
$$

Thus, the general solution of the homogeneous equation (41) has the form

$$
G_{11}=R^{-T} \Gamma R^{-1},
$$

with $\Gamma$ defined in (35). This, together with (40), gives rise to the general solution of (39)

$$
G_{11}=-R^{-T} \Gamma R^{-1}-M_{11} S+S^{T} M_{11} .
$$

Substituting (48) into (37) yields (v).

Sufficiency. From the description of (i)-(vi), we can obtain that (33) holds.

Remark 11. When $k=n$, by Theorem 10 , the solution of the Problem 1 with $D=0$ is given by

$$
M=Q M_{Q} Q^{T}, \quad G=Q G_{Q} Q^{T}, \quad K=Q K_{G} Q^{T},
$$

where

$$
\begin{gathered}
G_{\mathrm{Q}}=-R^{-T} \Gamma R^{-1}-M_{\mathrm{Q}} S+S^{T} M_{\mathrm{Q}}, \\
K_{\mathrm{Q}}=R^{-T} \Gamma \Lambda R^{-1}-S^{T} M_{\mathrm{Q}} S,
\end{gathered}
$$

with $M_{\mathrm{Q}}>0$ and $M_{\mathrm{Q}}=M_{\mathrm{Q}}^{T}$ which can be arbitrarily chosen.

\section{Particular Solution with $D=0$ and $K<0$}

In practice, the matrix $K$ in the Problem 1 with $D=0$ is sometimes required to be symmetric negative definite [5]. In this section, we will apply Theorem 10 to construct such a solution. We first prove the following lemma.

Lemma 12. For any given matrix defined in (35), we can construct a symmetric positive definite matrix $M_{11}$ so that $K_{11}$ defined in Theorem 10 is symmetric negative definite.

Proof. Since $S=R \Lambda R^{-1}$, it is easy to see that $K_{11}$ in Theorem 10 is symmetric negative definite if and only if the matrix

$$
\Gamma \Lambda-\Lambda^{T} R^{T} M_{11} R \Lambda
$$

is symmetric negative definite.

By the assumption (A2), we can first construct a symmetric positive definite matrix $\widetilde{M}$ so that $-\widetilde{M}+\Gamma \Lambda<0$. Then we use $\widetilde{M}$ to construct the desired $M_{11}$.

From (35) and (30), we denote

$$
\Gamma \Lambda=\operatorname{diag}\left\{\Gamma_{1} \Lambda_{1}, \ldots, \Gamma_{\ell_{3}} \Lambda_{\ell_{3}}\right\}
$$

with

$$
\begin{gathered}
\Gamma_{j} \Lambda_{j}=\left[\begin{array}{cccc}
0 & 0 & \theta_{j} & \omega_{j} \\
0 & 0 & -\omega_{j} & \theta_{j} \\
\theta_{j} & -\omega_{j} & 0 & 0 \\
\omega_{j} & \theta_{j} & 0 & 0
\end{array}\right], \\
\theta_{j}=-\alpha_{j} \xi_{j}-\beta_{j} \eta_{j} \quad j=1, \ldots, \ell_{1}, \\
\omega_{j}=-\alpha_{j} \eta_{j}+\beta_{j} \xi_{j}, \quad \\
\Gamma_{j} \Lambda_{j}=\left[\begin{array}{cc}
\theta_{j} & 0 \\
0 & \theta_{j}
\end{array}\right], \quad \theta_{j}=-\beta_{j} \eta_{j}, \quad j=\ell_{1}+1, \ldots, \ell_{2}, \\
\Gamma_{j} \Lambda_{j}=\left[\begin{array}{cc}
0 & \omega_{j} \\
\omega_{j} & 0
\end{array}\right], \quad \omega_{j}=-\alpha_{j} \eta_{j}, \quad j=\ell_{2}+1, \ldots, \ell_{3} .
\end{gathered}
$$

Here $\xi_{j}$ and $\eta_{j}$ are arbitrary real numbers. Take

$$
\widetilde{M}=\operatorname{diag}\left\{\widetilde{M}_{1}, \widetilde{M}_{2}, \ldots, \widetilde{M}_{\ell_{3}}\right\},
$$

with

$$
\begin{gathered}
\widetilde{M}_{j}=\left[\begin{array}{cccc}
x_{j} & z_{j} & \theta_{j} & \omega_{j} \\
z_{j} & y_{j} & -\omega_{j} & \theta_{j} \\
\theta_{j} & -\omega_{j} & a_{j} & c_{j} \\
\omega_{j} & \theta_{j} & c_{j} & b_{j}
\end{array}\right], \quad j=1, \ldots, \ell_{1}, \\
\widetilde{M}_{j}=\left[\begin{array}{cc}
x_{j} & 0 \\
0 & y_{j}
\end{array}\right], \quad j=\ell_{1}+1, \ldots, \ell_{2}, \\
\widetilde{M}_{j}=\left[\begin{array}{cc}
x_{j} & \omega_{j} \\
\omega_{j} & y_{j}
\end{array}\right], \quad j=\ell_{2}+1, \ldots, \ell_{3} .
\end{gathered}
$$


Using (53), if we choose $x_{j}, y_{j}, z_{j}, a_{j}, b_{j}$, and $c_{j}$ such that

$$
\begin{gathered}
x_{j}, y_{j}, a_{j}, b_{j}>0, \quad j=1, \ldots, \ell_{3}, \\
x_{j}>\left|z_{j}\right|+\left|\theta_{j}\right|+\left|\omega_{j}\right| \\
y_{j}>\left|z_{j}\right|+\left|\theta_{j}\right|+\left|\omega_{j}\right| \\
a_{j}>\left|c_{j}\right|+\left|\theta_{j}\right|+\left|\omega_{j}\right| \quad j=1, \ldots, \ell_{1}, \\
b_{j}>\left|c_{j}\right|+\left|\theta_{j}\right|+\left|\omega_{j}\right|, \\
x_{j}>\theta_{j} \\
y_{j}>\theta_{j}, \quad j=\ell_{1}+1, \ldots, \ell_{2}, \\
x_{j}>\left|\omega_{j}\right| \quad j=\ell_{2}+1, \ldots, \ell_{3}, \\
y_{j}>\left|\omega_{j}\right|, \quad
\end{gathered}
$$

then $\widetilde{M}>0$ and $\Gamma \Lambda-\widetilde{M}<0$. Obviously, such real numbers $x_{j}, y_{j}, z_{j}, a_{j}, b_{j}$, and $c_{j}$ can be easily chosen. Once $\widetilde{M}$ is determined, the required $M_{11}$ can be chosen by

$$
M_{11}=R^{-T} \Lambda^{-T} \widetilde{M} \Lambda^{-1} R^{-1} .
$$

Furthermore,

$$
K_{11}=R^{-T}(\Gamma \Lambda-\widetilde{M}) R^{-1}
$$

Using Lemma 12, we can construct a particular solution to the Problem 1 with $D=0, K<0$ as follows.

Algorithm 13. An algorithm for solving the Problem 1 with $D=0, K<0$ is proposed as follows.

(1) Input $\Lambda$ and $X$, compute the $Q R$ decomposition of $X$ according to (11), and compute $S=R \Lambda R^{-1}$.

(2) Choose $\Gamma$ as in (35) arbitrarily and compute $\Gamma \Lambda$ by (52) and (53).

(3) Construct a symmetric positive definite matrix $M_{11}$ by (54)-(57), compute $G_{11}$ by (iv), and compute $K_{11}$ by (v) in Theorem 10 or by (58).

(4) Choose arbitrary $M_{21}$ and $G_{21}$, and compute $K_{21}$ by (vi) in Theorem 10, $M_{12}=M_{21}^{T}$ and $G_{12}=-G_{21}^{T} K_{12}=$ $K_{21}^{T}$.

(5) Choose a symmetric positive definite matrix $\widehat{M} \epsilon$ $\mathbb{R}^{(n-k) \times(n-k)}$ and a symmetric negative definite matrix $\widehat{K} \in \mathbb{R}^{(n-k) \times(n-k)} ;$ compute $M_{22}=\widehat{M}+M_{21} M_{11}^{-1} M_{21}^{T}$, $K_{22}=\widehat{K}+K_{21} K_{11}^{-1} K_{21}^{T}$.

(6) Choose an arbitrary skew-symmetric matrix $G_{22}$, and form

$$
M=Q M_{Q} Q^{T}, \quad G=Q G_{Q} Q^{T}, \quad K=Q K_{Q} Q^{T},
$$

where $Q$ is given by (11).
Remark 14. When $k=n$, we only need to choose $\widetilde{M}$ by (54)(56), and compute $M_{Q}$ by (57), that is, $M_{11}$ is the whole $M_{Q}$; then use the same method described in Remark 8, we can obtain the particular solution of the Problem 1 with $D=0$ and $K<0$.

\section{Numerical Examples}

In this section, we present two numerical examples to illustrate the solutions constructed in Sections 2 and 4, respectively. For presentation, we report all numbers in 5 significant digits only, though all calculations are carried out in full precision.

Example 1. In this example, we use Algorithm 9 to construct the general solution of the Problem 1 . The partially prescribed eigeninformation $(\Lambda, X) \in \mathbb{R}^{4 \times 4} \times \mathbb{R}^{6 \times 4}$ as in (2)-(3) is given by the following eigenvalues and eigenvectors, which are from [12]:

$$
\begin{gathered}
\lambda_{1}=\bar{\lambda}_{2}=3.5121+8.2485 i, \quad \lambda_{3}=1.7541, \\
\lambda_{4}=1.2956,
\end{gathered}
$$

$$
\mathbf{x}_{1}=\overline{\mathbf{x}}_{2}=\left[\begin{array}{c}
9.2963+1.0480 i \\
2.3965+3.5650 i \\
3.8789+6.5809 i \\
2.8644+4.9742 i \\
1.5007+1.1356 i \\
1.9623+6.5805 i
\end{array}\right],
$$

$$
\mathbf{x}_{3}=\left[\begin{array}{l}
8.3476 \\
8.0946 \\
5.5542 \\
9.2809 \\
4.0705 \\
2.8111
\end{array}\right], \quad \mathbf{x}_{4}=\left[\begin{array}{l}
6.6044 \\
9.3147 \\
2.5443 \\
2.1294 \\
8.1057 \\
2.7021
\end{array}\right] \text {. }
$$

It is easy to check that the matrix pair $(\Lambda, X)$ satisfy the assumptions (A1) and (A2). According to Algorithm 9, by randomly choosing

$$
\begin{gathered}
M_{Q}=\operatorname{diag}\{1,2,5,4,3,6\}, \quad Z=\operatorname{diag}\{1,-1,1,-1\}, \\
C_{21}=\left[\begin{array}{llll}
8.3812 & 6.8128 & 8.3180 & 7.0947 \\
0.1964 & 3.7948 & 5.0281 & 4.2889
\end{array}\right], \\
C_{12}=\left[\begin{array}{ll}
3.0462 & 3.0276 \\
1.8965 & 5.4167 \\
1.9343 & 1.5087 \\
6.8222 & 6.9790
\end{array}\right], \\
C_{22}=\left[\begin{array}{ll}
3.7837 & 8.5366 \\
8.6001 & 5.9356
\end{array}\right], \quad K_{22}=\operatorname{diag}\{5,3\},
\end{gathered}
$$


we can figure out

$$
\begin{gathered}
M=\left[\begin{array}{cccccc}
1.4247 & -0.4938 & -0.5602 & -0.2909 & -0.2889 & 0.3442 \\
-0.4938 & 3.7424 & -0.2183 & 0.0056 & 0.4770 & -0.9513 \\
-0.5602 & -0.2183 & 4.2872 & -1.2080 & -0.4976 & -1.4334 \\
-0.2909 & 0.0056 & -1.2080 & 3.9308 & -0.0853 & -0.4537 \\
-0.2889 & 0.4770 & -0.4976 & -0.0853 & 3.5470 & -0.0537 \\
0.3442 & -0.9513 & -1.4334 & -0.4537 & -0.0537 & 4.0679
\end{array}\right], \\
D=\left[\begin{array}{ccccccc}
-16.1765 & -18.7748 & 18.4420 & -15.6724 & -16.2922 & 30.1788 \\
-18.7748 & 26.5196 & -11.6072 & 30.7981 & 9.2453 & -23.3017 \\
18.4420 & -11.6072 & 15.3413 & -23.9250 & -18.8430 & 12.6821 \\
-15.6724 & 30.7981 & -23.9250 & 24.9224 & 16.3251 & -29.7442 \\
-16.2922 & 9.2453 & -18.8430 & 16.3251 & 16.5213 & -8.0033 \\
30.1788 & -23.3017 & 12.6821 & -29.7442 & -8.0033 & 14.5419
\end{array}\right], \\
G=\left[\begin{array}{ccccccc}
0 & -30.6848 & 11.0912 & -25.4607 & -16.6322 & 16.4642 \\
30.6848 & 0 & 2.0333 & -4.7658 & 2.4935 & -4.0359 \\
-11.0912 & -2.0333 & 0 & -0.2491 & 1.5886 & 0.9319 \\
25.4607 & 4.7658 & 0.2491 & 0 & 5.0743 & -8.6382 \\
16.6322 & -2.4935 & -1.5886 & -5.0743 & 0 & -4.0057 \\
-16.4642 & 4.0359 & -0.9319 & 8.6382 & 4.0057 & 0
\end{array}\right], \\
K=\left[\begin{array}{ccccccc}
152.5248 & 60.1590 & 89.1902 & -73.6227 & -110.6742 & 70.6382 \\
60.1590 & -64.7406 & -67.6143 & -37.9090 & 15.3304 & -77.6697 \\
89.1902 & -67.6143 & 40.8772 & -18.4866 & 25.8457 & 9.9577 \\
-73.6227 & -37.9090 & -18.4866 & -1.2165 & 30.9425 & 15.3983 \\
-110.6742 & 15.3304 & 25.8457 & 30.9425 & 11.0952 & 50.7649 \\
70.6382 & -77.6697 & 9.9577 & 15.3983 & 50.7649 & -31.7764
\end{array}\right] .
\end{gathered}
$$

It is easy to check that $M$ is symmetric positive definite, $D$ and $K$ are symmetric, and $G$ is skew-symmetric. We define the residual as

$$
\operatorname{res}\left(\lambda_{j}, \mathbf{x}_{j}\right)=\left\|\left(\lambda_{j}^{2} M+\lambda_{j}(D+G)+K\right) \mathbf{x}_{j}\right\|_{2},
$$

and the numerical results are shown in Table 1. This shows that Algorithm 9 to construct the general solution of the Problem 1 is effective.

Example 2. In this example, we use Algorithm 13 to construct the general solution of the Problem 1 with $D=0$ and $K<0$. The partially prescribed eigeninformation $(\Lambda, X) \epsilon$ $\mathbb{R}^{8 \times 8} \times \mathbb{R}^{8 \times 8}$ as in (29)-(31) is given by randomly generated eigenvalues and eigenvectors

$$
\begin{gathered}
\lambda_{1}=\bar{\lambda}_{2}=0.2719+3.1269 i, \\
\lambda_{3}=\bar{\lambda}_{4}=-0.2719+3.1269 i, \\
\lambda_{5}=\bar{\lambda}_{6}=0+0.1286 i, \quad \lambda_{7}=-\lambda_{8}=3.8397,
\end{gathered}
$$

$$
\mathbf{x}_{1}=\overline{\mathbf{x}}_{2}=\left[\begin{array}{c}
6.8312+5.8692 i \\
0.9284+0.5758 i \\
0.3534+3.6757 i \\
6.1240+6.3145 i \\
6.0854+7.1763 i \\
0.1576+6.9267 i \\
0.1635+0.8408 i \\
1.9007+4.5436 i
\end{array}\right]
$$$$
\mathbf{x}_{3}=\overline{\mathbf{x}}_{4}=\left[\begin{array}{c}
4.4183+1.2105 i \\
3.5325+4.5075 i \\
1.5361+7.1588 i \\
6.7564+8.9284 i \\
6.9921+2.7310 i \\
7.2751+2.5477 i \\
4.7838+8.6560 i \\
5.5484+2.3235 i
\end{array}\right]
$$$$
\mathbf{x}_{5}=\overline{\mathbf{x}}_{6}=\left[\begin{array}{c}
8.0487+8.4387 i \\
9.0840+1.7390 i \\
2.3189+1.7079 i \\
2.3931+9.9430 i \\
0.4975+4.3979 i \\
0.7838+3.4005 i \\
6.4082+3.1422 i \\
1.9089+3.6508 i
\end{array}\right] \text {, }
$$ 


$$
\mathbf{x}_{7}=\left[\begin{array}{l}
3.9324 \\
5.9153 \\
1.1975 \\
0.3813 \\
4.5860 \\
8.6987 \\
9.3424 \\
2.6445
\end{array}\right], \quad \mathbf{x}_{8}=\left[\begin{array}{l}
1.6030 \\
8.7286 \\
2.3788 \\
6.4583 \\
9.6689 \\
6.6493 \\
8.7038 \\
0.0993
\end{array}\right]
$$

It is easy to check that the matrix pair $(\Lambda, X)$ satisfy the assumptions (A1) and (A2). According to Algorithm 13, by randomly choosing

$$
\Gamma=\operatorname{diag}\left\{\left[\begin{array}{cccc}
0 & 0 & 1.3701 & 8.1876 \\
0 & 0 & -8.1876 & 1.3701 \\
-1.3701 & 8.1876 & 0 & 0 \\
-8.1876 & -1.3701 & 0 & 0
\end{array}\right]\right.
$$

we can figure out

$$
\left.\left[\begin{array}{cc}
0 & 4.3017 \\
-4.3017 & 0
\end{array}\right],\left[\begin{array}{cc}
0 & 8.9032 \\
-8.9032 & 0
\end{array}\right]\right\} \text {, }
$$

and choosing

$$
\begin{aligned}
\widetilde{M}=\operatorname{diag}\{ & {\left[\begin{array}{cccc}
29.0320 & 0 & -25.9737 & 2.0583 \\
0 & 29.0320 & -2.0583 & -25.9737 \\
-25.9737 & -2.0583 & 29.0320 & 0 \\
2.0583 & -25.9737 & 0 & 29.0320
\end{array}\right], } \\
& {\left.\left[\begin{array}{cc}
0.4467 & 0 \\
0 & 0.4467
\end{array}\right],\left[\begin{array}{cc}
35.1854 & -34.1854 \\
-34.1854 & 35.1854
\end{array}\right]\right\}, }
\end{aligned}
$$

$$
\begin{aligned}
M & =\left[\begin{array}{cccccccc}
0.2381 & -0.0518 & -0.0448 & 0.1749 & -0.2085 & 0.2421 & -0.0454 & -0.4753 \\
-0.0518 & 0.7116 & 0.1091 & -0.0423 & -0.2386 & 0.0531 & -0.4977 & 0.3824 \\
-0.0448 & 0.1091 & 0.5424 & -0.6348 & 0.5762 & -0.5419 & -0.0180 & 0.4899 \\
0.1749 & -0.0423 & -0.6348 & 0.8628 & -0.8617 & 0.8260 & -0.1281 & -0.7994 \\
-0.2085 & -0.2386 & 0.5762 & -0.8617 & 0.9881 & -0.8915 & 0.3458 & 0.7424 \\
0.2421 & 0.0531 & -0.5419 & 0.8260 & -0.8915 & 1.0017 & -0.3058 & -0.9543 \\
-0.0454 & -0.4977 & -0.0180 & -0.1281 & 0.3458 & -0.3058 & 0.4810 & -0.0001 \\
-0.4753 & 0.3824 & 0.4899 & -0.7994 & 0.7424 & -0.9543 & -0.0001 & 1.4299
\end{array}\right], \\
G & =\left[\begin{array}{cccccccc}
0.0000 & -0.4167 & 0.0642 & -0.1869 & 0.3993 & -0.7528 & 0.7498 & 0.1786 \\
0.4167 & 0.0000 & 0.8272 & -0.5230 & 0.3188 & 0.2073 & -0.5834 & -0.4186 \\
-0.0642 & -0.8272 & -0.0000 & -0.4048 & 0.8542 & -1.1750 & 1.0802 & 0.6606 \\
0.1869 & 0.5230 & 0.4048 & -0.0000 & -0.3391 & 0.5388 & -0.7985 & -0.2182 \\
-0.3993 & -0.3188 & -0.8542 & 0.3391 & -0.0000 & -0.4504 & 0.7544 & 0.4519 \\
0.7528 & -0.2073 & 1.1750 & -0.5388 & 0.4504 & -0.0000 & -0.3404 & -0.9403 \\
-0.7498 & 0.5834 & -1.0802 & 0.7985 & -0.7544 & 0.3404 & -0.0000 & 0.6307 \\
-0.1786 & 0.4186 & -0.6606 & 0.2182 & -0.4519 & 0.9403 & -0.6307 & -0.0000
\end{array}\right], \\
K= & {\left[\begin{array}{ccccccccc}
-0.9602 & 1.0594 & -0.7478 & 0.6664 & -0.3476 & -0.0708 & -0.6132 & 1.2458 \\
1.0594 & -2.2568 & 0.5473 & -1.3328 & 1.1498 & -1.3154 & 2.2446 & -0.0867 \\
-0.7478 & 0.5473 & -1.7993 & 0.4842 & -0.1183 & -0.4577 & 0.2942 & 1.3384 \\
0.6664 & -1.3328 & 0.4842 & -1.0279 & 0.9632 & -0.9383 & 1.2189 & 0.2852 \\
-0.3476 & 1.1498 & -0.1183 & 0.9632 & -1.5571 & 1.7068 & -1.3474 & -0.8193 \\
-0.0708 & -1.3154 & -0.4577 & -0.9383 & 1.7068 & -2.7058 & 1.9965 & 2.2604 \\
-0.6132 & 2.2446 & 0.2942 & 1.2189 & -1.3474 & 1.9965 & -2.8310 & -0.9034 \\
1.2458 & -0.0867 & 1.3384 & 0.2852 & -0.8193 & 2.2604 & -0.9034 & -4.5291
\end{array}\right] . }
\end{aligned}
$$

It is easy to check that $M$ is symmetric positive definite, $K$ is symmetric negative definite, and $G$ is skew-symmetric. We define the residual as

$$
\operatorname{res}\left(\lambda_{j}, \mathbf{x}_{j}\right)=\left\|\left(\lambda_{j}^{2} M+\lambda_{j} G+K\right) \mathbf{x}_{j}\right\|_{2},
$$

and the numerical results are shown in Table 2. This shows that Algorithm 13 to construct the particular solution of the Problem 1 with $D=0$ and $K<0$ is effective.

\section{Conclusions}

In this paper, we first use techniques involving matrix decompositions to derive an expression of the general solution to the question, for a set of given $(k \leq n)$ pairs of complex numbers and vectors (closed under conjugation), under assumptions (A1) and (A2). Then, with the special properties $D=0$ and $K<0$, we construct a particular solution. Numerical results illustrate these solutions. 
TABLE 1: Example 1.

\begin{tabular}{lcccc}
\hline$\left(\lambda_{j}, x_{j}\right)$ & $\left(\lambda_{1}, x_{1}\right)$ & $\left(\lambda_{2}, x_{2}\right)$ & $\left(\lambda_{3}, x_{3}\right)$ & $\left(\lambda_{4}, x_{4}\right)$ \\
\hline $\operatorname{res}\left(\lambda_{j}, x_{j}\right)$ & $1.7708 e-012$ & $1.7708 e-012$ & $5.8528 e-013$ & $7.2918 e-013$ \\
\hline
\end{tabular}

TABLE 2: Example 2.

\begin{tabular}{lcccccccc}
\hline$\left(\lambda_{j}, x_{j}\right)$ & $\left(\lambda_{1}, x_{1}\right)$ & $\left(\lambda_{2}, x_{2}\right)$ & $\left(\lambda_{3}, x_{3}\right)$ & $\left(\lambda_{4}, x_{4}\right)$ & $\left(\lambda_{5}, x_{5}\right)$ & $\left(\lambda_{6}, x_{6}\right)$ & $\left(\lambda_{7}, x_{7}\right)$ & $\left(\lambda_{8}, x_{8}\right)$ \\
\hline $\operatorname{res}\left(\lambda_{j}, x_{j}\right)$ & $4.3271 e-014$ & $4.3271 e-014$ & $4.9065 e-014$ & $4.9065 e-014$ & $1.0209 e-014$ & $1.0209 e-014$ & $7.3644 e-014$ & $6.9718 e-014$ \\
\hline
\end{tabular}

For another case of $k>n$, it is rather complex, and the proof method in Theorem 5 seems not to be used directly to find a solution of Problem 1. Fortunately, for the damped nongyroscopic system, Cai et al. [13] solved the QIEP with $2 n \geq k>n$ given eigenpairs. However, case $k>n$ has never been discussed in the literature for damped gyroscopic system. It might be an interesting research and needs further investigation.

\section{Conflict of Interests}

The authors declare that there is no conflict of interests regarding the publication of this paper.

\section{Acknowledgment}

Guo-Liang Chen is supported by the National Natural Science Foundation of China (no. 11071079).

\section{References}

[1] M. J. Balas, "Trends in large space structure control theory: fondest hopes, wildest dreams," IEEE Transactions on Automatic Control, vol. 27, no. 3, pp. 522-535, 1982.

[2] Z. Bai and Y. Su, "Soar: a second-order arnoldi method for the solution of the quadratic eigenvalue problem," SIAM Journal on Matrix Analysis and Applications, vol. 26, no. 3, pp. 640-659, 2005.

[3] C. Guo, "Numerical solution of a quadratic eigenvalue problem," Linear Algebra and Its Applications, vol. 385, no. 1-3, pp. 391-406, 2004.

[4] Z. Jia and Y. Sun, "A refined Jacobi-Davidson method for the quadratic eigenvalue problem," in Proceedings of the 10th WSEAS International Confenrence on APPLIED MATHEMATICS, pp. 1150-3155, Dallas, Tex, USA, November 2006.

[5] K. Meerbergen, "The quadratic arnoldi method for the solution of the quadratic eigenvalue problem," SIAM Journal on Matrix Analysis and Applications, vol. 30, no. 4, pp. 1463-1482, 2008.

[6] J. Qian and W. Lin, "A numerical method for quadratic eigenvalue problems of gyroscopic systems," Journal of Sound and Vibration, vol. 306, no. 1-2, pp. 284-296, 2007.

[7] F. Tisseur and K. Meerbergen, "The quadratic eigenvalue problem," SIAM Review, vol. 43, no. 2, pp. 235-286, 2001.

[8] L. Zhou, L. Bao, Y. Lin, Y. Wei, and Q. Wu, "Restarted generalized second-order krylov subspace methods for solving quadratic eigenvalue problems," World Academy of Science, Engineering and Technology, vol. 67, pp. 429-436, 2010.
[9] I. Gohberg, P. Lancaster, and L. Rodman, "On selfadjoint matrix polynomials," Integral Equations and Operator Theory, vol. 2, no. 3, pp. 434-439, 1979.

[10] M. Chu and S. Xu, "Spectral decomposition of real symmetric quadratic $\lambda$-matrices and its applications," Mathematics of Computation, vol. 78, no. 265, pp. 293-313, 2009.

[11] Z. Jia and M. Wei, "A real-valued spectral decomposition of the undamped gyroscopic system with applications," SIAM Journal on Matrix Analysis and Applications, vol. 32, no. 2, pp. 584-604, 2011.

[12] Y. Kuo, W. Lin, and S. Xu, "Solutions of the partially described inverse quadratic eigenvalue problem," SIAM Journal on Matrix Analysis and Applications, vol. 29, no. 1, pp. 33-53, 2006.

[13] Y. Cai, Y. Kuo, W. Lin, and S. Xu, "Solutions to a quadratic inverse eigenvalue problem," Linear Algebra and Its Applications, vol. 430, no. 5-6, pp. 1590-1606, 2009.

[14] Y. Yuan, "An inverse eigenvalue problem for damped gyroscopic second-order systems," Mathematical Problems in Engineering, vol. 2009, Article ID 725616, 10 pages, 2009.

[15] H. W. Braden, “The equations $A^{T} X \pm X^{T} A=B$," Journal on Matrix Analysis and Applications, vol. 20, no. 2, pp. 295-302, 1999. 


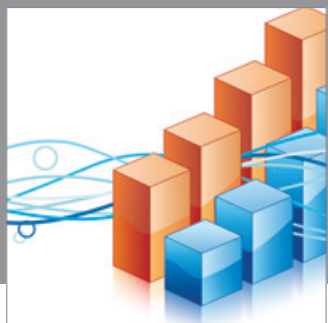

Advances in

Operations Research

mansans

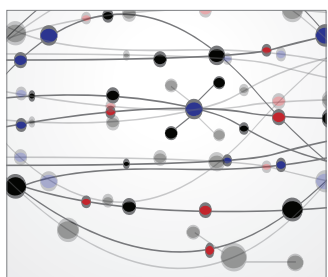

The Scientific World Journal
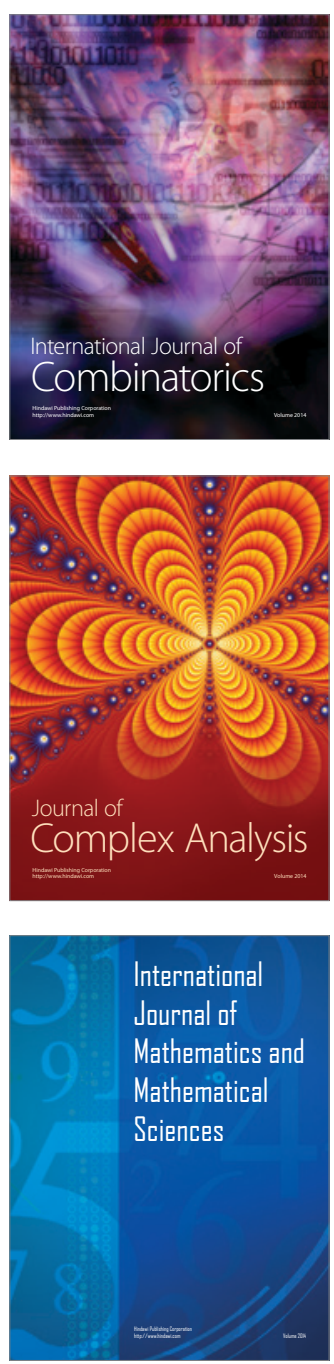
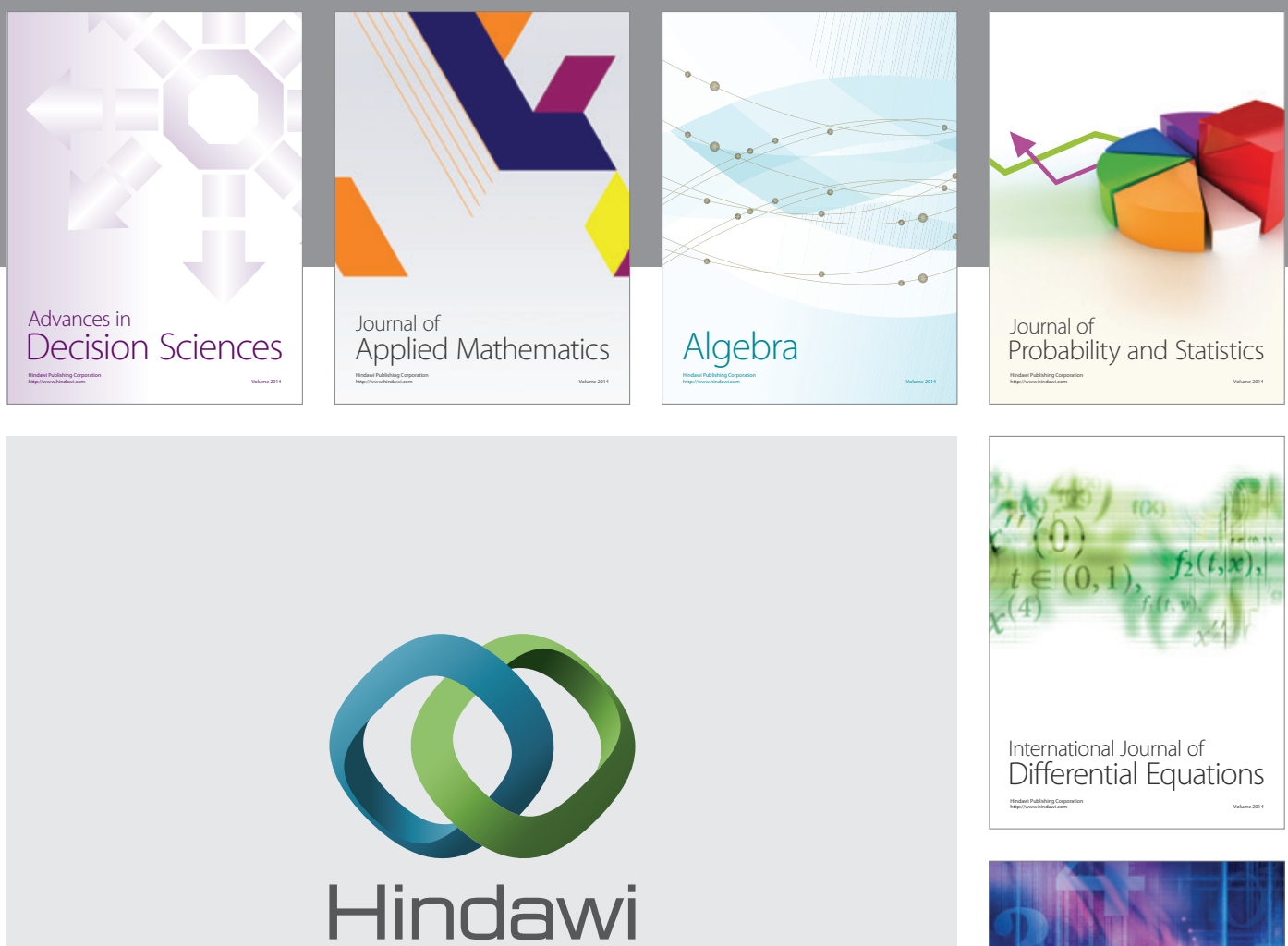

Submit your manuscripts at http://www.hindawi.com
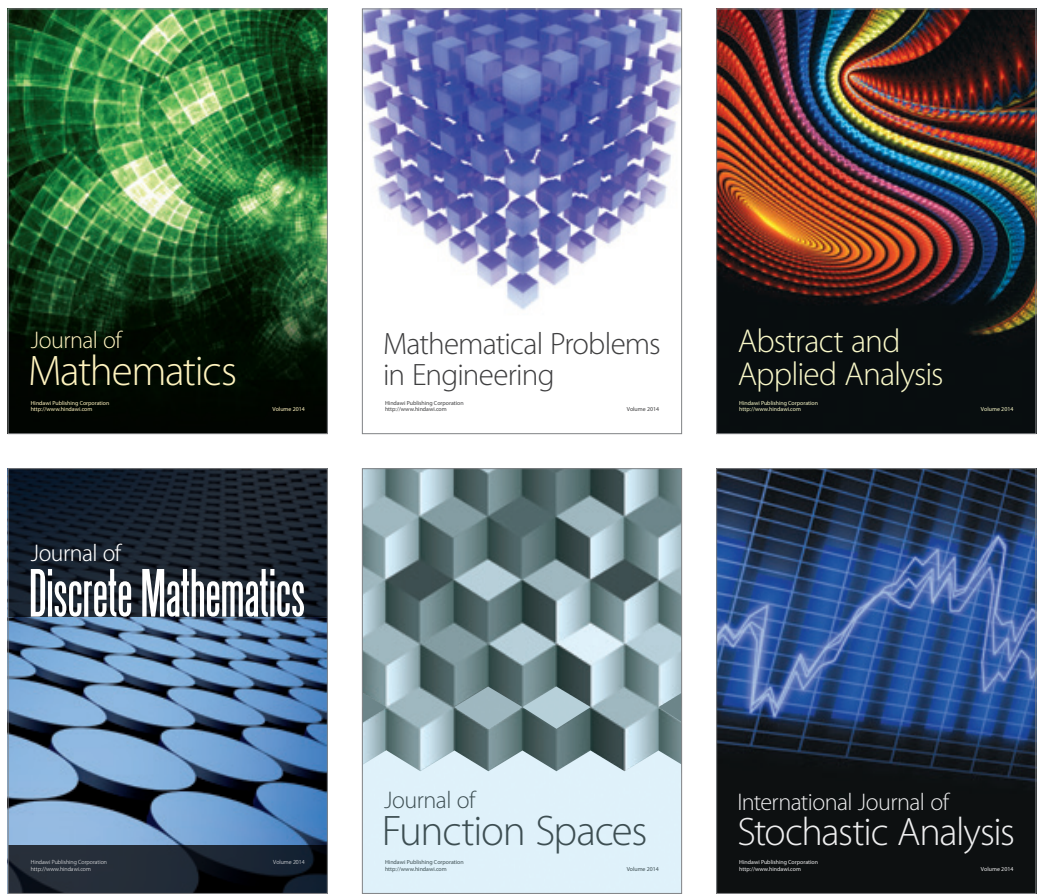

Journal of

Function Spaces

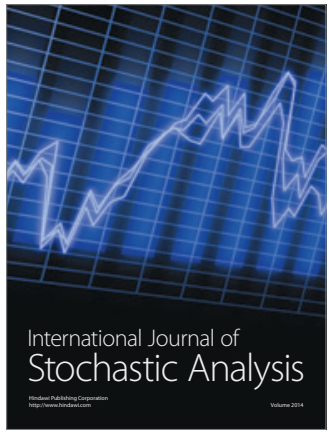

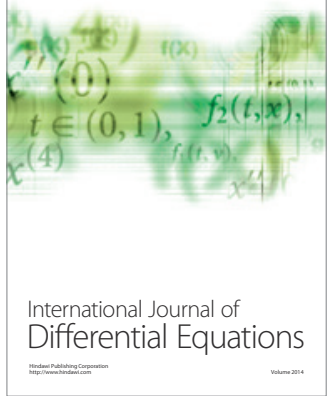
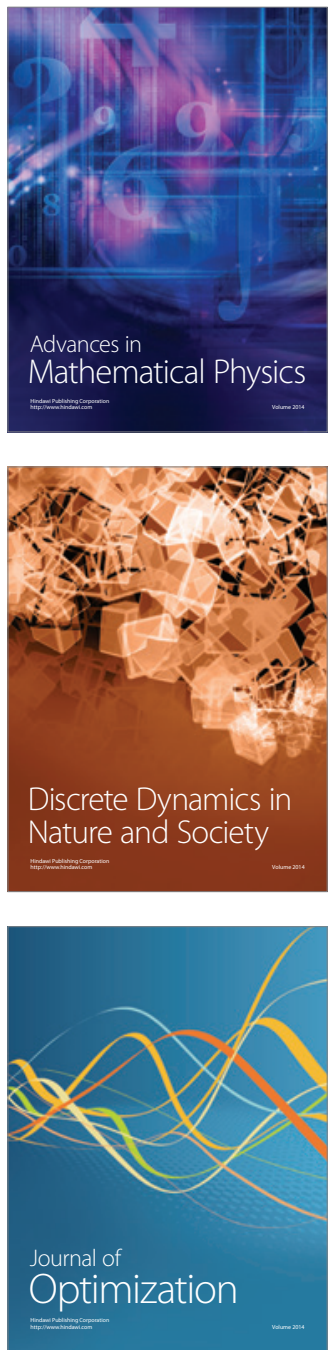